\title{
Potential Effects of Hormone Therapy in Type 2 Idiopathic Macular Telangiectasia
}

\author{
Ute E.K. Wolf-Schnurrbusch ${ }^{a}$ Irene Leung ${ }^{b}$ Ferenc B. Sallob, d \\ Traci E. Clemons ${ }^{e}$ Emily Y. Chew ${ }^{f}$ Alan C. Bird ${ }^{c}$ Tunde Peto $^{g, h}$ \\ MacTel Study Group \\ a Universtätsklinik für Augenheilkunde, University of Bern, Bern, Switzerland; ${ }^{\mathrm{b}}$ Department of Research and \\ Development and 'Inherited Eye Disease, Moorfields Eye Hospital NHS Foundation Trust, and d UCL Institute of \\ Ophthalmology, London, UK; ${ }^{\mathrm{e} E M M E S}$ Corporation, Washington, DC, and ${ }^{\mathrm{f}}$ National Eye Institute, National Institutes \\ of Health, Bethesda, MD, USA; ${ }^{9}$ Queen's University Belfast Faculty of Medicine Health and Life Sciences, Belfast, and \\ ${ }^{h}$ NIHR Biomedical Research Centre for Ophthalmology, Moorfields Eye Hospital NHS Foundation Trust and UCL \\ Institute of Ophthalmology, London, UK
}

\section{Keywords}

Idiopathic macular telangiectasia - MacTel Study · Neuroprotection · Estrogen · Tamoxifen

\begin{abstract}
Purpose: To investigate the influence of hormone therapy with tamoxifen or estrogens on morphological changes in macular telangiectasia (MacTel) type 2 patients as revealed clinically in multiple imaging modalities. Methods: Patients with a history of tamoxifen or estrogen use were selected from the cohort of the MacTel Study. A race-, age- and bestcorrected visual acuity-matched group of MacTel participants not under hormone therapy served as the comparison group. The frequencies of typical features of the MacTel phenotype apparent in color fundus, red-free, fluorescein angiographic and optical coherence tomographic images were graded and analyzed statistically. Results: Thirty-nine MacTel patients were included in the analyses, of whom 13 were receiving tamoxifen, 13 estrogens and 13 patients no hormone treatment. Patients treated with estrogens showed
\end{abstract}

\section{KARGER}

(C) 2017 S. Karger AG, Basel

E-Mail karger@karger.com

www.karger.com/ore significantly fewer breaks in the ellipsoid zone on optical coherence tomography ( 7 eyes, $29.1 \%$, vs. tamoxifen: 14 eyes, $53.8 \%$, and vs. controls: 14 eyes, $53.8 \%, p=0.04$ in both analyses, Fisher exact test). Retinal crystalline deposits were significantly more frequent in patients receiving estrogens (12 eyes, $16.2 \%$, vs. 2 eyes, $2.7 \%, p=0.003$, Fisher exact test). No significant between-group differences were apparent with regard to other features of the phenotype (extent of retinal low reflective spaces, late hyperfluorescence on fluorescein angiography or retinal thickness). Conclusions: Tamoxifen treatment does not seem to accentuate structural changes in patients with MacTel type 2. Treatment with estrogens may exhibit a neuroprotective effect as suggested by the decreased frequency of ellipsoid zone breaks in corresponding patients, although corroborative studies are warranted to confirm these exploratory data.

(c) 2017 S. Karger AG, Basel

The list of all participating principal investigators and centers in the MacTel Study can be found in the Appendix.

Ferenc B. Sallo, MD, PhD

The Reading Centre, Department of Research and Development

Moorfields Eye Hospital NHS Foundation Trust

162 City Road, London EC1V 2PD (UK)

E-Mail Ferenc.Sallo@ moorfields.nhs.uk 


\section{Introduction}

Idiopathic macular telangiectasia (MacTel) type 2 is a bilateral, progressive retinal disease potentially leading to a loss of central vision [1]. The pathogenesis and natural history of the disease have so far been poorly understood. Historically considered a vascular disease, more recent clinical and experimental evidence points to a hereditary cause and a neurodegenerative pathogenesis in which retinal Müller cells may play a central role [1-3].

Clinically, MacTel is characterized by vascular signs comprising telangiectatic vessels, dilated, right-angle veins, fluorescein leakage in the angiogram and potentially secondary sub/intraretinal neovascularization $[4$, $5]$, and by neurodegenerative signs including the presence of refractile crystals in the inner retina [6], a loss of retinal transparency in the parafoveal region (also exhibiting increased reflectivity to short wavelength light), midretinal hyperpigmented plaques, low-reflective intraretinal spaces, and a progressive outer retinal atrophy identified on optical coherence tomography (OCT) images as a break in the line attributed to the ellipsoid zone (EZ) of the photoreceptors $[1,7]$.

Evidence of the role of estrogens in neurodegenerative disease has steadily increased in recent years. Estrogenmediated neuroprotective mechanisms in the retina have been suggested $[8,9]$, though evidence of the role of estrogens in the retina remains limited to date.

Tamoxifen is a type II antiestrogen with mixed estrogenic/antiestrogenic properties. It is used as adjuvant therapy for estrogen-dependent breast carcinoma. Ocular toxicity from tamoxifen is well documented and characterized by bilateral superficial refractile deposits in the inner layers of the retina as well as foveal cystoid spaces apparent on OCT [10]. These features resemble characteristics observed in MacTel.

The role of concomitant hormone therapy in influencing the natural history of MacTel type 2 has not been investigated so far. The purpose of the present study is to investigate the possible influence of hormone therapy (tamoxifen or estrogens) on clinical morphological changes in MacTel type 2 patients.

\section{Materials and Methods}

Patients were selected from the cohort of the MacTel Study, an international multicenter prospective study of the phenotype and potential therapies of MacTel, based on a history of past or present use of tamoxifen or estrogen hormone replacement therapy. Age-, race- and best-corrected visual acuity-matched MacTel type 2 pa- tients not receiving hormone therapy were selected for a control group. Eyes with neovascularization or secondary fibrotic scars were excluded.

Baseline characteristics and eligibility criteria for enrolment into the study have been published previously [11]. The study protocol adhered to the tenets of the Declaration of Helsinki and was approved by the local institutional review/ethics committee of each participating site. Prior to enrolment in the study, written, informed consent was obtained from each participant following an explanation of the nature of the study.

Medical and ocular history and lifestyle information were collected using standardized questionnaires. Multimodal images including stereoscopic color/red-free fundus photographs and fluorescein angiograms were recorded digitally. Color and fluorescein angiography images were analyzed for the presence of characteristics of MacTel (loss of retinal transparency, telangiectatic vessels, right-angled venules, retinal crystals and fluorescein leakage/staining) using image overlays of a grading grid according to the International Classification for ARM/AMD protocol as described previously $[11,12]$.

OCT was performed using Stratus TD-OCT devices (Carl Zeiss Meditec, Dublin, CA, USA). High-quality crosshair scans (signal strength $>7$ ) were analyzed for the presence, size, and location relative to the foveal center of inner and outer retinal low-reflective spaces, for the presence of an enlarged foveal depression, for the presence of a break in the EZ and for pigment migration into the midretina. Retinal thickness was assessed by subfield in scans acquired using the "fast macular" thickness protocol.

Statistical analyses were performed using GraphPad Prism version 5.00 for Windows (GraphPad Software, San Diego, CA, USA). Demographic characteristics for the patients were summarized using descriptive statistics expressed in terms of means \pm standard deviation or proportions. Between-group analyses for all clinical and imaging parameters were performed by 2 -way analysis of variance for continuous and by the Fisher exact test for categorical variables. A $p$ value of 0.05 or less was accepted as statistically significant.

\section{Results}

A total of 39 patients (78 eyes) were included in these analyses, characteristics of each group are presented in Table 1 . Four out of the 78 eyes were excluded, 2 having fibrotic scars $(2.6 \%)$ and the other 2 subretinal neovascularization (2.6\%).

Crystalline deposits were seen significantly more often in patients receiving estrogens than in those not receiving estrogens ( 12 eyes, $16.2 \%$, vs. 2 eyes, $2.7 \%, p=0.003$, Fisher exact test). Patients receiving tamoxifen also demonstrated more crystals than controls but the difference did not reach statistical significance ( 8 eyes, $10.8 \%, p=0.072$, Fisher exact test). Crystals were seen at the level of the internal limiting membrane, outside the central subfield of the International Classification (IC) grid. Differences between groups in loss of retinal transparency, visible telan-
2

Ophthalmic Res

DOI: $10.1159 / 000479932$
Wolf-Schnurrbusch et al. 
Table 1. Distribution by age, gender, and visual acuity of patients included in the analyses

\begin{tabular}{cccc}
\hline & Tamoxifen & $\begin{array}{l}\text { Estrogen } \\
\text { HRT }\end{array}$ & Control \\
& & $13(26)$ & $13(26)$ \\
\hline $\begin{array}{l}\text { Patients (eyes), } n \\
\text { Gender (female/male) }\end{array}$ & $11 / 2$ & $13 / 0$ & $11 / 2$ \\
$\begin{array}{c}\text { Age at baseline, years } \\
\text { Mean } \pm \text { SD }\end{array}$ & $65.3 \pm 11.4$ & $65.1 \pm 5.5$ & $64.3 \pm 8.6$ \\
$\quad$ Range & $50-75$ & $54-75$ & $51-75$ \\
$\begin{array}{c}\text { BCVA (letter score) } \\
\text { Right eyes }\end{array}$ & & & \\
$\quad \begin{array}{l}\text { Mean } \pm \text { SD } \\
\text { Range }\end{array}$ & $70.5 \pm 7.2$ & $68.5 \pm 7.9$ & $67.5 \pm 11.8$ \\
$\quad 39-86$ & $50-78$ & $42-87$ \\
$\quad$ Left eyes & & & \\
$\quad \begin{array}{l}\text { Mean } \pm \text { SD } \\
\text { Range }\end{array}$ & $69.0 \pm 11.4$ & $68.4 \pm 10.0$ & $67.7 \pm 107$ \\
Worse eye & $50-88$ & $44-81$ & $50-84$ \\
$\quad$ Mean \pm SD & $63.5 \pm 11.6$ & $63.2 \pm 8.3$ & $61.7 \pm 8.7$ \\
$\quad$ Range & $39-86$ & $44-72$ & $42-75$ \\
\hline
\end{tabular}

giectatic vessels, or hyperfluorescence on fluorescein angiography were not statistically significant.

Patients treated with estrogens showed EZ breaks less frequently compared with either other group (7 eyes, $29.1 \%$, vs. tamoxifen: 14 eyes, $53.8 \%$, and vs. controls: 14 eyes, $53.8 \%, p=0.04$ in both analyses, Fisher exact test). No statistically significant differences were demonstrable in any other OCT characteristic investigated.

\section{Discussion}

Initially, MacTel was considered a vascular disease; however, over the last few years it has become evident that photoreceptor loss is integral to the disease and it may now be considered a disorder of neuroglial origin $[2,4$, 13]. Conceivably the vascular changes may be secondary to neuroglial disease, as has been recorded in animal models of photoreceptor degeneration and humans with retinitis pigmentosa [5-10]. In recent years a compelling case has been put forward for the use of estrogens as protective agents against neurodegenerative disorders of the central nervous system $[14,15]$. Their role in influencing synaptic plasticity, brain development and memory is well established. Several studies have highlighted the potential neuroprotective action of estrogens against damage produced by acute and chronic injury to the adult brain [16-18]. The cellular and molecular mechanisms

Potential Effects of Hormone Therapy in

Type 2 Macular Telangiectasia implicated in these neuroprotective effects however appear to be complex [19-21].

Controversy exists as to the precise role of estrogens that can both afford neuroprotection and precipitate ischemic cerebral incidents $[22,23]$.

The potential of estrogens in providing neuroprotection to the retina is less well understood. Bovine and rat retinas have been shown to express estrogen receptors $\alpha$ and $\beta$ throughout the retinal thickness [19-21, 24, 25], and estrogens have shown promise in influencing the natural history of retinitis pigmentosa, age-related macular degeneration and glaucoma as well as exercising neuroprotective properties against ischemia-reperfusion injury in the context of transient retinal ischemia [26-28].

On the other hand, tamoxifen has long been identified as causative of a crystalline retinopathy exhibiting macular cystoid changes on OCT distinct from those seen in cystoid macular edema [10]. The pathogenesis of tamoxifen-induced retinal toxicity is unclear, it may act in vivo as an antagonist of glutamate transporters in retinal pigment epithelial cells, as already demonstrated in vitro [29]. A presumed increase in glutamate could explain the axonal degeneration observed in histopathology specimens and the formation of crystalline deposits. Müller cell impairment may follow retinal neuron injury resulting in atrophy and the formation of the optically empty spaces observed on OCT in the foveal area [30]. On the other hand, it has been suggested that tamoxifen could afford a potential neuroprotective effect through, among other modes of action, free radical scavenging. The mixed estrogenic/antiestrogenic properties of tamoxifen could potentially explain its seemingly contradictory actions of neuroretinal toxicity and neuroprotective effect.

The present study in its exploratory nature aims to investigate the possibility of a link between hormone therapy and any of the typical findings encountered on imaging in patients with MacTel type 2. The significantly lower frequency of EZ breaks visible on OCT in estrogentreated patients points to a potential beneficial effect of estrogens in reducing the rate of progression in MacTel type 2. A break in the EZ has close functional correlates, and increase in its area is an established measure of disease progression in MacTel [31]. The presence of EZ breaks is clearly detectable even using TD-OCT that was available in the initial years of the MacTel Study, prior to the introduction of spectral domain technology.

Crystalline deposits and the appearance of macular cystoid spaces on OCT are strikingly similar in both tamoxifen retinopathy and MacTel type 2. In this study, 
however no increased severity of imaging findings in patients with MacTel type 2 receiving tamoxifen could be identified rendering unlikely the possibility of cumulative retinal toxicity in these cases. The significance of increased prevalence of crystalline deposits in patients receiving estrogens is rather difficult to interpret.

A reasonable criticism on the use of natural estrogens as neuroprotectants relates to their feminizing properties that would be undesirable to male patients. The use of synthetic estrogen analogs would however circumvent such limitations allowing benefiting from their antioxidant properties regardless of gender or health predispositions.

Limitations of the present study reside in its exploratory nature, limited sample size, and the lack of randomization. Our findings are however encouraging and warrant further research to accrue information on the precise role of hormone therapy on the natural history of $\mathrm{MacTel}$ type 2.

\section{Acknowledgment}

The authors wish to thank the Lowy Medical Research Institute (LMRI) and the NIHR for providing support for funding this study. The LMRI also participated in the design of the study and in the review and approval of the manuscript.

\section{Disclosure Statement}

All authors declare that there is no conflict of interest.

\section{Appendix}

Participating Principal Investigators and Centers in the MacTel Study

Jose-Alain Sahel, MD, PhD, Centre Hopitalier National d'Ophtalmologie des Quinze-Vingts, Paris, France; Robyn Guymer, MD, Centre for Eye Research, East Melbourne, Australia; Gisele Soubrane, MD, PhD, FEBO, Clinique d'Ophtalmologie de Créteil, Créteil, France; Alain Gaudric, MD, Hôpital Lariboisière, Paris, France; Steven Schwartz, MD, Jules Stein Eye Institute, UCLA, Los Angeles, CA, USA; Ian Constable, MD, Lions Eye Institute, Nedlands, WA, Australia; Michael Cooney, MD, MBA, Manhattan Eye, Ear, and Throat Hospital, New York, NY, USA; Catherine Egan, MD, Moorfields Eye Hospital, London, UK; Lawrence Singerman, MD, Retina Associates of Cleveland, Cleveland, $\mathrm{OH}$, USA; Mark C. Gillies, MD, PhD, Save Sight Institute, Sydney, NSW, Australia; Martin Friedlander, MD, PhD, Scripps Research Institute, La Jolla, CA, USA; Daniel Pauleikhoff, Prof. Dr., St. Franziskus Hospital, Muenster, Germany; Joseph Moisseiev, MD, Goldschleger Eye Institute, Tel Hashomer, Israel; Richard Rosen, MD, New York Eye and Ear Infirmary, New York, NY, USA; Robert Murphy, MD, Retina Group of Washington, Fairfax, VA, USA; Frank Holz, MD, University of Bonn, Bonn, Germany; Grant Comer, MD, University of Michigan, Kellogg Eye Center, Ann Arbor, MI, USA; Barbara Blodi, MD, University of Wisconsin, Madison, WI, USA; Diana Do, MD, Wilmer Eye Institute, Baltimore, MD, USA; Alexander Brucker, MD, Scheie Eye Institute, Philadelphia, PA, USA; Raja Narayanan, MD, LV Prasad Eye Institute, Hyderabad, India; Sebastian Wolf, MD, PhD, University of Bern, Bern, Switzerland; Philip Rosenfeld, MD, PhD, Bascom Palmer, Miami, FL, USA; Paul S. Bernstein, MD, PhD, Moran Eye Center, University of Utah, UT, USA; Joan W. Miller, MD, Massachusetts Eye and Ear Infirmary, Harvard Medical School, Boston, MA, USA; Lawrence Yannuzzi, MD, Vitreous, Retina, Macula Consultants of New York, NY, USA; Jacque Duncan, MD, University of California, San Francisco, CA, USA; Mina Chung, MD, University of Rochester Medical Center, Rochester, NY, USA; Jiong Yan, MD, Emory University, Atlanta, GA, USA; David Weinberg, MD, Medical College of Wisconsin, Milwaukee, WI, USA; Clasien Oomen, MD, Radboud University Medical Center, Nijmegen, The Netherlands.

\section{References}

1 Charbel Issa P, et al: Macular telangiectasia type 2. Prog Retin Eye Res 2013;34:49-77.

2 Powner MB, et al: Loss of Muller's cells and photoreceptors in macular telangiectasia type 2. Ophthalmology 2013;120:2344-2352.

3 Powner MB, et al: Perifoveal Müller cell depletion in a case of macular telangiectasia type 2. Ophthalmology 2010;117:2407-2416.

4 Gass JD, Oyakawa RT: Idiopathic juxtafoveolar retinal telangiectasis. Arch Ophthalmol 1982;100:769-780.

5 Gass JD, Blodi BA: Idiopathic juxtafoveolar retinal telangiectasis. Update of classification and follow-up study. Ophthalmology 1993; 100:1536-1546.
6 Sallo FB, et al: Retinal crystals in type 2 idiopathic macular telangiectasia. Ophthalmology $2011 ; 118: 2461-2467$.

7 Gaudric A, et al: Optical coherence tomography in group $2 \mathrm{~A}$ idiopathic juxtafoveolar retinal telangiectasis. Arch Ophthalmol 2006; 124:1410-1419.

8 Nirmalan PK, et al: Female reproductive factors and eye disease in a rural South Indian population: the Aravind Comprehensive Eye Survey. Invest Ophthalmol Vis Sci 2004;45: 4273-4276.

9 Hayashi Y, et al: Neuroprotective effect of 17beta-estradiol against N-methyl-D-aspartate-induced retinal neurotoxicity via p-ERK induction. J Neurosci Res 2007;85:386-394.
10 Gualino V, et al: Optical coherence tomography findings in tamoxifen retinopathy. Am J Ophthalmol 2005;140:757-758.

11 Clemons TE, et al: Baseline characteristics of participants in the natural history study of macular telangiectasia (MacTel) MacTel Project Report No 2. Ophthalmic Epidemiol 2010;17:66-73.

12 Bird AC, et al: An international classification and grading system for age-related maculopathy and age-related macular degeneration. The International ARM Epidemiological Study Group. Surv Ophthalmol 1995;39:367374. 
13 Powner MB, et al: Perifoveal muller cell depletion in a case of macular telangiectasia type 2 . Ophthalmology 2010;117:2407-2416.

14 Engler-Chiurazzi EB, et al: Estrogens as neuroprotectants: estrogenic actions in the context of cognitive aging and brain injury. Prog Neurobiol 2016, Epub ahead of print.

15 Engler-Chiurazzi EB, Singh M, Simpkins JW: From the 90s to now: a brief historical perspective on more than two decades of estrogen neuroprotection. Brain Res 2016;1633: 96-100.

16 Wakade C, et al: Tamoxifen neuroprotection in cerebral ischemia involves attenuation of kinase activation and superoxide production and potentiation of mitochondrial superoxide dismutase. Endocrinology 2008;149:367379.

17 Zhang Y, et al: Neuroprotection by tamoxifen in focal cerebral ischemia is not mediated by an agonist action at estrogen receptors but is associated with antioxidant activity. Exp Neurol 2007;204:819-827.

18 Biewenga E, Cabell L, Audesirk T: Estradiol and raloxifene protect cultured SN4741 neurons against oxidative stress. Neurosci Lett 2005;373:179-183.
19 Amantea D, et al: From clinical evidence to molecular mechanisms underlying neuroprotection afforded by estrogens. Pharmacol Res 2005;52:119-132.

20 Marin R, et al: Estrogen activates classical and alternative mechanisms to orchestrate neuroprotection. Curr Neurovasc Res 2005;2:287301.

21 Hall JM, Couse JF, Korach KS: The multifaceted mechanisms of estradiol and estrogen receptor signaling. J Biol Chem 2001;276: 36869-36872.

22 Kimelberg HK: Tamoxifen as a powerful neuroprotectant in experimental stroke and implications for human stroke therapy. Recent Pat CNS Drug Discov 2008;3:104-108.

23 Zhang H, et al: Tamoxifen mediated estrogen receptor activation protects against early impairment of hippocampal neuron excitability in an oxygen/glucose deprivation brain slice ischemia model. Brain Res 2009;1247:196211.

24 Kobayashi K, et al: Estrogen receptor expression in bovine and rat retinas. Invest Ophthalmol Vis Sci 1998;39:2105-2110.
25 Kumar DM, et al: Role of nonfeminizing estrogen analogues in neuroprotection of rat retinal ganglion cells against glutamate-induced cytotoxicity. Free Radic Biol Med 2005; 38:1152-1163.

26 Dykens JA, et al: Photoreceptor preservation in the S334ter model of retinitis pigmentosa by a novel estradiol analog. Biochem Pharmacol 2004;68:1971-1984.

27 Newman-Casey PA, et al: The potential association between postmenopausal hormone use and primary open-angle glaucoma. JAMA Ophthalmol 2014;132:298-303.

28 Hutchinson CV, Walker JA, Davidson C: Oestrogen, ocular function and low-level vision: a review. J Endocrinol 2014;223:R9R18.

29 Maenpaa $\mathrm{H}$, et al: Glutamate uptake is inhibited by tamoxifen and toremifene in cultured retinal pigment epithelial cells. Pharmacol Toxicol 2002;91:116-122.

30 Dyer MA, Cepko CL: Control of Muller glial cell proliferation and activation following retinal injury. Nat Neurosci 2000;3:873-880.

31 Sallo FB, et al: The IS/OS junction layer in the natural history of type 2 idiopathic macular telangiectasia. Invest Ophthalmol Vis Sci 2012;53:7889-7895.
Potential Effects of Hormone Therapy in

Type 2 Macular Telangiectasia
Ophthalmic Res 